\title{
Teenage sleep and technology engagement across the week
}

\author{
Amy Orben ${ }^{\text {Corresp., } 1,2}$, Andrew K Przybylski ${ }^{3,4}$ \\ 1 Emmanuel College, University of Cambridge, Cambridge, United Kingdom \\ 2 \\ 2 MRC Cognition and Brain Sciences Unit, University of Cambridge, Cambridge, United Kingdom \\ 3 Department of Experimental Psychology, University of Oxford, Oxford, United Kingdom \\ 4 Oxford Internet Institute, University of Oxford, Oxford, United Kingdom \\ Corresponding Author: Amy Orben \\ Email address: aco35@cam.ac.uk
}

Background. Throughout the developed world, adolescents are growing up with increased access to and engagement with a range of screen-based technologies, allowing them to encounter ideas and people on a global scale from the intimacy of their bedroom. The concerns about digital technologies negatively influencing sleep are therefore especially noteworthy, as sleep has been proven to greatly affect both cognitive and emotional well-being. The associations between digital engagement and adolescent sleep should therefore be carefully investigated in research adhering to the highest methodological standards. This understood, studies published to date have not often done so and have instead focused mainly on data derived from general retrospective self-report questionnaires. The value of this work has been called into question by recent research showing that retrospective questionnaires might fail to accurately measure these variables of interest. Novel and diverse approaches to measurement are therefore necessary for academic study to progress.

Methods. This study analyses data from 11,884 adolescents included in the UK Millennium Cohort Study to examine the association between digital engagement and adolescent sleep, comparing the relative effects of retrospective self-report versus time-use diary measures of technology use. By doing so, it provides an empirical lens to understand the effects of digital engagement both throughout the day and before bedtime and adds nuance to a research area primarily relying on retrospective self-report.

Results. The study finds that there is a small negative association relating digital engagement to adolescent sleep both on weekdays and weekend days (median standardized association $\beta_{\text {weekday }}=-0.06$ and $\beta_{\text {weekend }}=-0.03$ ). There is a more negative association between digital engagement and total sleep time on weekdays compared to weekend days (median standardized $\beta_{\text {weekday }}=-0.08$, median standardized $b_{\text {weekend }}=-0.01$ ), while there is no such difference when examining adolescents' bedtime. Surprisingly, and contrary to our expectations, digital technology use before bedtime is not significantly associated with the amount of sleep and the tardiness of bedtime in adolescents.

Conclusions. Results derived from the use of transparent Specification Curve Analysis methods show that the negative associations in evidence are mainly driven by retrospective technology use measures and measures of total time spent on digital devices during the day. The effects are overall very small: for example an additional hour of digital screen time per day was only related to a 9-minute decrease in total time spent sleeping on weekdays and a 3-minute decrease on weekends. Using digital screens 30 minutes before bed led to a 1-minute decrease in total time spent sleeping on weekdays and weekends. The study shows that more work should be done examining how to measure digital screen time before interventions are designed. 


\section{Teenage sleep and technology engagement across}

\section{2 the week}

3

4

5 Amy Orben ${ }^{1,2}$, Andrew K. Przybylski ${ }^{3,4}$

6

$7 \quad{ }^{1}$ Emmanuel College, University of Cambridge, Cambridge, UK

$8{ }^{2}$ MRC Cognition and Brain Sciences Unit, University of Cambridge, Cambridge, UK

$9{ }^{3}$ Department of Experimental Psychology, University of Oxford, Oxford, UK

$10{ }^{4}$ Oxford Internet Institute, University of Oxford, Oxford, UK

11

12 Corresponding Author:

13 Amy Orben ${ }^{1,2}$

14 Emmanuel College, Cambridge, CB2 3AP, UK.

15 Email address: aco35@cam.ac.uk

16 


\section{Abstract}

18

19

20

21

22

23

24

25

26

27

28

29

30

31

32

33

34

35

36

37

38

39

40

41

42

43

44

45

46

47

48

49

50

51

52

53

54

55

56

Background. Throughout the developed world, adolescents are growing up with increased access to and engagement with a range of screen-based technologies, allowing them to encounter ideas and people on a global scale from the intimacy of their bedroom. The concerns about digital technologies negatively influencing sleep are therefore especially noteworthy, as sleep has been proven to greatly affect both cognitive and emotional well-being. The associations between adolescents' digital engagement and sleep should therefore be carefully investigated in research adhering to the highest methodological standards. This understood, studies published to date have not often done so; instead focusing mainly on data derived from general retrospective selfreport questionnaires. The value of this approach has been called into question by recent studies showing that retrospective questionnaires might fail to accurately measure these variables of interest. Novel and diverse approaches to measurement are therefore necessary for academic study to progress.

Methods. This study analyses data from 11,884 adolescents included in the UK Millennium Cohort Study to examine the association between digital engagement and adolescent sleep, comparing the relative effects of retrospective self-report versus time-use diary measures of technology use. The effects of digital engagement throughout the day and before bedtime were evaluated using Specification Curve Analysis with the aim of adding critical nuance to a research area primarily relying on retrospective self-report.

Results. The study finds that there is a small negative association relating digital engagement to adolescent sleep both on weekdays and weekend days (median standardized association $\beta_{\text {weekday }}$ $=-0.06$ and $\beta_{\text {weekend }}=-0.03$ ). There is a more negative association between digital engagement and total sleep time on weekdays compared to weekend days (median standardized $\beta_{\text {weekday }}=$ 0.08 , median standardized $\beta_{\text {weekend }}=-0.02$ ), while there is no such difference when examining adolescents' bedtime. Surprisingly, and contrary to our expectations, there was no clear trend demonstrating that digital technology use before bedtime was associated with either the amount of sleep or a delay of bedtime in adolescents.

Conclusions. Results show that the negative associations in evidence are mainly driven by retrospective technology use measures and measures of total time spent on digital devices during the day. The effects are overall very small: for example an additional hour of digital screen time per day was only related to a 9-minute decrease in total time spent sleeping on weekdays and a 3minute decrease on weekends. Using digital screens 30 minutes before bed was associated with a 1-minute decrease in total time spent sleeping on weekdays and weekends. The study highlights the need for higher quality measures of digital screen time before interventions are designed.

\section{Introduction}


57

58

59

60

61

62

63

64

65

66

67

68

69

70

71

72

73

74

75

76

77

78

79

80

81

82

83

84

85

86

87

88

89

90

91

92

93

94

95

96

97

British children now spend an average of 2 hours and 11 minutes each day online, using a diverse range of smartphone, tablet, and computer screens (Ofcom, 2019). This engagement oftentimes continues past bedtime: research shows that nearly two thirds $(62 \%)$ of children aged 12-15 are granted permission by their caretakers to take their mobile phone to bed (Ofcom, 2019). Given this emerging norm, it is an understandable concern that digital screen engagement might impact both sleep quantity and quality (Owens, 2014). Yet it is not clear whether this common-sense perception is rooted in scientific evidence. Approximately two thirds of British children believe that they have "a good balance between screen time and doing other things", and therefore seem to think that they are not adversely affected by the ever-increasing presence of digital screens in their lives (Ofcom, 2019). This apparent divergence in views raises the question of whether commonly held apprehensions about digital screen engagement are empirically justified and whether the persistent use of digital screens really impairs sleep habits.

This larger question is growing into an increasingly pressing concern for both scientists and policymakers in various developed countries (Davies, Atherton, Calderwood, \& McBride, 2019; Viner, Davie, \& Firth, 2019). The notion that digital screens have a consistent and negative effect on sleep is indeed worrying, especially as sleep has been identified as a key factor in adolescent development (Owens, 2014; Scott, Biello, \& Woods, 2019; Woods \& Scott, 2016), impacting both executive function and emotional stability (Beebe, 2011; Owens, 2014). A loss of sleep resulting from digital device use should therefore be a topic of conversation for academics, parents, and policy makers alike, as they are stakeholders who would need to collaborate closely and rapidly to find solutions to this potential problem (Woods \& Scott, 2016).

There are many mechanisms by which digital technology engagement could forestall sleep onset and drive decreases in adolescent sleep more broadly. Device use may simply displace time that could be spent sleeping - one cannot be sleeping and simultaneously scrolling through Instagram - though the directionality of this association is still unclear (Levenson, Shensa, Sidani, Colditz, \& Primack, 2017; Owens, 2014; Przybylski, 2018). Digital engagement might facilitate a cascade of behaviors such as socializing which replace time sleeping (Scott, Biello, \& Cleland, 2018), or use might simply be physiologically stimulating to the extent it delays sleep onset (Cain \& Gradisar, 2010), or elicits subjective emotional experiences like fear of missing out which provide tantalizing alternatives to sleep (Scott et al., 2018). A growing body of correlational and experimental research has linked digital screen use before bed to negative effects (Harbard, Allen, Trinder, \& Bei, 2016; Levenson et al., 2017; Orzech, Grandner, Roane, $\&$ Carskadon, 2016) and has postulated that this could be due to later bed times (Cain \& Gradisar, 2010; Orzech et al., 2016) or decreased relaxation (Harbard et al., 2016). Taken together, there are multiple plausible mechanisms linking digital screen use, decreasing sleep and later bedtimes, but little clarity about whether these exist and, if they do, which theoretical explanation gives the most accurate representation of ultimate causal links. 
A possible reason for the lack of consistent and substantial evidence for linkages between digital engagement and adolescent sleep is the low quality of measurement available in current data sources. The extant literature examining adolescents' use of digital technologies relies, in many cases, on operationalizations of 'screen time': a somewhat nebulous and questionable construct (Orben, Etchells, \& Przybylski, 2018; Orben \& Przybylski, 2019a). The challenges associated with using 'screen time' as a predictive variable is exacerbated by the use of retrospective selfreport measures which require adolescents to estimate and report how much digital technology they engage with. Human participants in research are inherently bad at judging how much time they spend on a wide variety of activities, and 'screen time' is no exception (Boase \& Ling, 2013; Schwarz \& Oyserman, 2001). An expanding body of research has made it abundantly clear that asking a teenager to estimate the amount of time they have allocated to digital screens is not an optimal measurement strategy (Robinson, 1985; Scharkow, 2016). Furthermore, inaccuracies in reporting are influenced by the intensity of a participants' screen time, creating a systematic bias which is difficult to adjust for statistically (Vanden Abeele, Beullens, \& Roe, 2013; Wonneberger \& Irazoqui, 2017). Improved, diverse and convergent measurement approaches to gauge digital engagement are therefore necessary to ultimately obtain robust and replicable insights into the effect of this activity on adolescents and their sleep.

One promising alternative to retrospective self-report measures are time-use diaries: a measurement method where participants keep a structured journal about what they are doing throughout one or two study days. Broadly speaking, time-use diaries are a form of experience sampling methodology: a research approach which has been used to probe a wide range of analogue pursuits including well-being, motivation, and relationship processes (Reis, Gable, \& Maniaci, 2013) and, more recently, digital technology use (Masur, 2019; Verduyn et al., 2015). More specifically, time-use diaries require adolescents to fill out what they were doing on a fixed interval or task-contingent basis, and provide a record of what kinds of activities a young person engages with during any given time window of their day (Ipsos MORI, 2016). If adolescents report engaging in digital activities, the diaries can be used to calculate whether participants used digital devices at all, how much time they spent using digital devices and when in the day these devices were used.

The empirical value of time-use diary measurements has been increasingly acknowledged by social science researchers who analyze and collect such data. These approaches have now been included in high-quality large cohort studies such as Growing Up in Ireland and the US Panel Study of Income Dynamics (Williams et al., 2009). Although this method falls short of the 'perfect world' of direct behavioral tracking (see: Andrews, Ellis, Shaw, \& Piwek, 2015; David, Roberts, \& Christenson, 2018), it represents a clear diversification of current practices and is not associated with the technical and ethical difficulties of installing software on the devices used by young people (Constine, 2019). Although this method comes with its own limitations, it introduces a more diverse picture of digital technology use than is present in the academic 
138 literature. Further, it can aide researchers disambiguating the effects of different levels of

139

140

141

142

143

144

145

146

147

148

149

150

151

152

153

154

155

156

157

158

159

160

161

162

163

164

165

166

167

168

169

170

171

172

173

174

175

176

177

engagement and effects arising from engagement at different times of day - for example before bedtime. In a policy landscape where parents and caregivers are increasingly advised to limit adolescent digital engagement on the basis of research done using retrospective self-report data, diversifying measurement by including time-use diary measurements is an increasingly exciting scientific prospect.

\section{The Present Research}

In this study we investigated the association between digital screen engagement and adolescent sleep using a unique high-quality birth cohort dataset that includes both retrospective self-report measures of digital engagement, time-use diary data, as well as sleep duration and bedtimes data. We used these data to create additional measures of bedtimes and digital technology use both throughout the day and in the 30-minute period before bedtime. Furthermore, both the retrospective self-report and diary measures were available for weekdays and weekend days separately. Briefly, our study aimed to explore three research questions: first, how does digital technology use relate to sleep onset and duration on weekdays? Second, How does digital technology use relate to sleep onset and duration on weekends? And third, How is the relationship between digital technology use and sleep affected by the measurement practices used to quantify digital technology use?

To fully explore these relations arising from our research questions we apply Specification Curve Analysis (SCA; Simonsohn, Simmons, \& Nelson, 2015). This analytical method allows us to determine the extent to which the availability, use, and time of use of digital technologies impacts teen's sleep outcomes, while taking into account the range of possible analytical decisions we could have made, and the analytical pathways we could have taken to ultimately analyze the data. Our goal is to provide a transparent overview of the effects found in the data available, supporting robust inferences regarding the nature of the relations between digital technology use and adolescent sleep.

\section{Materials \& Methods}

\section{Dataset}

This research data used in this study was drawn from the Millennium Cohort Study (MCS), a nationally representative longitudinal birth cohort study which tracks a cohort of young people as they live their lives in the United Kingdom (University of London, Institute for Education, 2017). The sample includes young people who were born between September 2000 and January 2001 and the sampling frame oversamples minorities and those from disadvantaged backgrounds to allow for direct comparisons between those who do and do not suffer material deprivations. We did not use weightings in our analyses. Furthermore, we did not impute missing data and instead used listwise deletion. The MCS data we analyzed in this study originated from two

Peer) reviewing PDF | (2019:07:39889:2:0:NEW 12 Dec 2019) 
178 aspects of the project. First, retrospective data was extracted from the omnibus self-report survey 179 which was administered in 2015 and 2016; A total of 11,884 adolescents (5,931 girls and 5,953 180 boys) completed this aspect of the study. Their ages ranged from 2,864 13-year-olds, to 8,860 181 14-year-olds and 160 15-year-olds. Second, we used data from the time-use diaries which 4,642 182 adolescents, a subsample of the omnibus participants, completed. Not all participants could fill 183 out the time use diaries due to restrictions in the amount of activity monitors available that were

184

185

186

187

188

189

190

191

192

193

194

195

196

197

198

199

200

201

202

203

204

205

206

207

208

209

210

211

212

213

214

215

216

217 administered concurrently, but whose data we do not evaluate in this study (Ipsos MORI, 2016). In other words, the subsample of those who completed time-use diary data is about one third of the sample available for analyses of only retrospective self-report data. The participants who completed time-use diary data differ significantly from those who did not in terms of age ( $m_{\text {not }}$ completed $\left.=13.79, m_{\text {completed }}=13.75\right)$, gender $\left(m_{\text {not completed }}=0.53, m_{\text {completed }}=0.45\right)$, closeness to parents ( $\left.m_{\text {not completed }}=3.20, m_{\text {completed }}=3.23\right)$, retrospectively-reported screen time $\left(m_{\text {not completed }}=\right.$ $\left.4.85, m_{\text {completed }}=4.76\right)$ but not self-reported sleep duration on weekends ( $m_{\text {not completed }}=10.55$, $\left.m_{\text {completed }}=10.51\right)$ and weekdays $\left(m_{\text {not completed }}=8.61, m_{\text {completed }}=8.64\right)$. The participants who completed the time use diaries were more likely to be younger, female, closer to their parents and use less screens.

\section{Ethical Review}

Ethical approval for the MCS was given by the U.K. National Health Service (NHS) London, Northern, Yorkshire and South-West Research Ethics Committees (MREC/01/6/19, MREC/03/2/022, 05/MRE02/46, 07/MRE03/32). Parents gave written consent, while adolescents provided oral consent.

\section{Measures}

In this study we considered measures of bedtime derived from time use diaries and retrospective self-report questionnaires, in addition to measures of sleep duration and sleep difficulties derived solely from retrospective self-report questionnaires. Likewise, we made full use of the available technology engagement data collected in both the retrospective and time-use components of the MCS.

The time-use diaries were developed specifically for the MCS by Ipsos MORI and the Centre for Time Research at the University of Oxford and included two 24-hour time windows (weekday and weekend) where the cohort members reported on what they are doing throughout the day (Ipsos MORI, 2016). They were administered in the 10 days after the interviewer visit where the adolescent filled out the questionnaires and were available on the web, through an app or on paper. The web- and paper-based versions were designed in such a way that participants reported on 10-minute intervals filled out throughout the day from 4am to 4am the next day. On the app, however, they could drag and drop activities up to a minute's interval for that time frame. When recording their activities, the adolescents were provided with a wide variety of 44 activity codes to choose from; one code related to sleep, while five codes related to digital engagement, we will 
218 detail these codes in the time-use diary sections below. The time-use diaries could also be filled 219 in retrospectively, e.g. noting sleep times.

220

221

222

223

224

225

226

227

228

229

230

231

232

233

234

235

236

237

238

239

240

241

242

243

244

245

246

247

248

249

250

251

252

253

254

255

256

257

Retrospective self-reports of sleep. In our main analyses we examined measures of bedtime and sleep duration on weekend days and weekdays using retrospective self-report questionnaires. In additional analyses we also examined sleep difficulties. Bedtime on weekdays was measured by a self-report question which asked "About what time do you usually go to sleep on a school night?", for weekend days it asked "About what time do you usually go to sleep on the nights when you do not have school the next day?": 1 = "before 9pm", 2 = "9-9:59pm", 3 = "1010:59pm", 4 = "11-midnight", 5 = "after midnight". These scores were reversed so that higher scores mean earlier bedtimes. The mean for bedtime on weekdays was $3.06(\mathrm{sd}=0.96)$ and 2.04 $(\mathrm{sd}=0.93)$ on weekends. While these measures were on a 5-point scale, we treated them as continuous because it was important for us to apply the same analytical approach to all specifications (analytical pathways) applied in this research.

To calculate total time spent sleeping on weekend days or weekdays we took into account the reported wake up times. Wake up times were measured on weekdays using the following question: "About what time do you usually wake up in the morning on a school day?", $1=$ "before 6am", 2 = "6-6:59am", 3 = "7-7:59am", 4 = "8-8:59am", 5 = "after 9am". On a weekend day, wake up time were measured using the question: "About what time do you wake up in the morning on the days when you do not have school?", 1 = "before 8am", 2 = "8-8:59am", 3 = "9-9:59am", 4 = "10-10:59am", 5 = "11-11:59am", 6 = "after Midday". To obtain the total time sleeping we first calculated the approximate bedtime: adding 7.5 to the adolescent's score on the retrospective self-report bedtime question. This meant, for example, that a score of 1 ("before 9pm") became 8.5, and a score of 4 ("11-midnight") became 11.5. We therefore assumed the middle of the range selected to be the bedtime to be the actual bedtime. We calculated the amount of time spent sleeping before midnight by subtracting this bedtime value from 12. For example, for those who scored 4 ("11-midnight"), we coded their bedtime to be 11.5 , and therefore their total time spent sleeping before midnight was 0.5 .

We then calculated the time spent sleeping after midnight: for weekdays we added 4.5 to the score on the self-report wake up time question and for weekends we added 6.5 to the score on the self-report wake up time question. Again this assumed the middle of the range selected to be the wake up time. We then summed the time spent sleeping before and after midnight for both weekend and weekdays separately and used the measure as continuous. For the measure of total time spent sleeping on weekdays the mean was $8.62(\mathrm{sd}=1.04)$ and on weekends it was 10.53 $(\mathrm{sd}=1.23)$.

Furthermore, we also examined sleep difficulties in additional analyses. These difficulties were measured using two retrospective self-report scales concerning how long it takes for an 
258

259

260

261

262

263

264

265

266

267

268

269

270

271

272

273

274

275

276

277

278

279

280

281

282

283

284

285

286

287

288

289

290

291

292

293

294

295

296

297

adolescent to fall asleep and whether the adolescent wakes up during the night. In our regressions we analyzed the variables as continuous. The former is measured by asking: "During the last four weeks, how long did it usually take for you to fall asleep?, $1=$ "0-15 minutes", 2 = "16-30 minutes", 3 = "31-45 minutes", 4 = "46-60 minutes", 5 = "more than 60 minutes" (mean = 3.73 , sd $=1.27$ ). The latter is measured by asking: "During the last four weeks, how often did you awaken during your sleep time and have trouble falling back to sleep again?", 1 = "all of the time", 2 = "most of the time", 3 = "a good bit of the time", 4 = "some of the time", $5=$ "a little of the time", and $6=$ "none of the time" $($ mean $=4.61, \mathrm{sd}=1.37)$.

Time-use diary reports of sleep. We also measured adolescents' bedtime using their time-use diaries, where adolescents could indicate that they were sleeping during certain times using a specific code (they would need to fill out those areas of the time use diary retrospectively). To locate when the adolescent went to sleep in the evening we wrote a piece of code that scanned through each adolescent's time use diary and noted the last time during their specific record when their activity changed from a non-sleep activity to sleep. As the time use diary ranged from 4am on the study day to $4 \mathrm{am}$ on the next day this should provide us accurate bedtimes for most adolescents (i.e. those who went to bed before $4 \mathrm{am}$ ). For those adolescents who did not have any sleep onset during the diary day (i.e. they went to bed after $4 a m$ the next day), we assumed they went to sleep at $4 \mathrm{am}$. We did not include any measure of sleep duration, as the estimation of this using the same day's wakeup time would have required making assumptions that we found untenable. For example, if the teenager filled the time-use diary out on a Monday, and we wanted to measure sleep duration we would only have information for their wake-up time on Monday morning and bedtime on Monday evening. We would therefore need to assume that their wake-up time on Monday morning would be identical to their wake-up time on Tuesday morning, and then use this to calculate the time between their bedtime on Monday evening and their wake-up time on Tuesday morning. As wake-up times are subject to change, we did not think the assumption that Monday and Tuesday wake-up times are always equal would be beneficial to this study.

The scale of these measures was on a continuous scale from 1 to 144 (10 minute intervals throughout the 24-hour day, with higher scores meaning earlier in the day). The mean for the time gone to sleep was $30.22(\sim 11 \mathrm{pm}$, standard deviation 14.41$)$ on a weekday and $27.74(\sim$ 11:20pm, standard deviation 13.81) on a weekend.

Retrospective reports of digital engagement. The dataset included a retrospective self-report digital screen engagement item set which asked: "On a normal week during term time, how many hours do you spend... ", a) "watching television programmes or films? Please remember to include time spent watching programmes or films on a computer or mobile device as well as on a $T V$, DVD etc. Please also include time spent before school as well as time after school" b) "playing electronic games on a computer of games systems, such as Wii, Nintendo D-S, X-Box or 
298

299

300

301

302

303

304

305

306

307

308

309

310

311

312

313

314

315

316

317

318

319

320

321

322

323

324

325

326

327

328

329

330

331

332

333

334

335

336

337

PlayStation? Please remember to include time before school as well as time after school?" c) "using the internet? Remember to include time spent using the internet on tablets, Smartphones and other mobile devices as well as computers and laptops. Please don't include time spent using the internet at school, but remember to include time before and after school and anytime for homework", and d) "on social networking on messaging sites or Apps on the internet such as Facebook, Twitter and WhatsApp?". For all four questions the response options ranged from $1=$ "none", 2 = "less than half an hour", 3 = "half an hour to less than 1 hour", 4 = "1 hour to less than 2 hours", $5=$ "2 hours to less than 3 hours", $6=$ "3 hours to less than 5 hours", $7=$ " 5 hours to less than 7 hours", to $8=$ "7 hours or more". To obtain a composite digital screen engagement items we took the measures' means, deleting those adolescents who had missing values in one or more of the four items. The mean of the scores on the composite measure was 4.82 while the standard deviation was 1.26 . The measure was treated as continuous.

Time-use diaries of digital engagement. Digital technology use was also measured in the timeuse diaries as adolescents could use five different codes to signify time spent using technologies: "answering emails, instant messaging, texting", "browsing and updating social networking sites", "general internet browsing, programming", "playing electronic games and Apps" and "watching TV, DVDs, downloaded videos". These five codes were aggregated to form the digital engagement score, which was then split into three distinct measures of digital technology use: (1) Participation - whether the adolescent engaged in any digital screen engagement during the day, (2) Time spent - for those adolescents who did participate in digital screen engagement, we measured the total time spent on such activities, and (3) Bedtime technology use - whether digital technology was reported to be used 30 minutes before the bedtime reported in the timeuse diaries. Like with the sleep measures, these measurements were separated for weekdays and weekend days. For the dichotomous participation measure on weekdays the mean was 0.81 and the standard deviation 0.39 ; on weekends the mean was 0.85 and the standard deviation 0.35 . For the continuous time spent measure on weekdays the mean was 3.45 and the standard deviation 2.49, while on weekends the mean was 4.59 and the standard deviation 2.98. Lastly, for bedtime technology use on weekdays the mean was $0.46(\mathrm{sd}=0.50)$ and on weekends the mean was 0.47 (sd 0.50).

We bifurcated time-use diary measurements into participation and time spent because the raw values showed high positive skew. In other words, while many participants did not register engaging in any digital technology use throughout the day, very few participants registered a very large amount of digital technology use. In accordance to previous studies (Orben \& Przybylski, 2019a; Rohrer \& Lucas, 2018), we therefore split the time use variable into participation and time spent (Hammer, 2012; Rohrer \& Lucas, 2018). For amount of technology use before bed we chose to use a dichotomous measure examining 30 minutes before bedtime because this would include only proximal technology use before bed. We could have also included longer before-bed timeframes (e.g. 1 or 2 hours) or used continuous measures of how 
338

339

340

341

342

343

344

345

346

347

348

349

350

351

352

353

354

355

356

357

358

359

360

361

362

363

364

365

366

367

368

369

370

371

372

373

374

375

376

377

much technology was used in such longer timeframes. We decided against this because of the binned nature of the time-use diaries. That said, we acknowledge that such choices could have been included in a more elaborate specification curve model.

Covariates and confounding variables. We included a variety of covariates in the analysis of this data, having chosen these on the basis of existing theory and past studies (Orben \& Przybylski, 2019b; Parkes, Sweeting, Wight, \& Henderson, 2013; Przybylski, 2018). The control variables spanned maternal, family, child and demographic factors. Firstly, the demographic factors included the sex and age of the child and the ethnicity of the mother. Sex was coded dichotomously with 1 being male and 0 being female, the age of the child was a continuous variable and the ethnicity of the mother was coded as a factor, white (1) and non-white (0), to assess the ethnic majority/minority. We did not take child ethnicity, because we were concerned about the quality of responding as the participants were asked about ethnicity at a young age. We decided parent ethnicity would be a more consistent and high-quality measure.

Next the family-level control variables included weekly family income, whether the father lives with the family and the number of siblings in household. These are important to control for as the family environment could causally affect both sleep and screen habits. Weekly family income and number of siblings in household were continuous measures provided by the parent while the adolescents were asked whether their father lives with them, which was coded as a dichotomous variable (no $=0$, yes $=1$ ). The mother-level control variables included parent word activity score, highest academic qualification, time spent with child and the caregiver's score on the Kessler depression scale. These control variables were included to account for the home environment and parenting, which could both influence screen use and sleep. The word activity score is a measure of cognitive capacity and provides a continuous score from 0 to 20 . The highest academic qualification was measured from National Vocational Qualification Level 1 to 5 , we coded foreign qualifications or those who answered "none of these" as NA: it was coded as a factor. Time spent with child was measured on a 5-level continuous scale: "too much time", "more than enough time", "just enough time", "not quite enough time", nowhere near enough time". The final family-level factor, parent scores on the Kessler depression scale were included, ranged from 0 to 24 , and was treated as a continuous control variable.

Lastly, additional child-level control variables included closeness to parents, long-term illness and educational motivation. These items are included to attempt to control for both parenting and other factors that could influence either of the variables of interest. We included long-term illness, and no other well-being scores, because we wanted to account for debilitating aspects in the child's life but felt that general well-being items could be mediators of the relationship between screen use and sleep. Closeness to parents was measured with four four-item questions asking how close the child was with both mother and father and also how often they argued with mother and father respectively. If the child reported not having a father or mother, we coded

Peer) reviewing PDF | (2019:07:39889:2:0:NEW 12 Dec 2019) 
378 them as NA. Educational motivation was measured using a six item questionnaire asking "How

379 often do you try your best at school", "How often do you find school interesting", "How often do 380 you feel unhappy at school", "How often do you get tired at school", "How often do you feel 381 school is a waste of time", "How often difficult to keep mind on work at school". The two first 382 questions were reversed and the mean of the six items were taken: those with missing values for 383 any of the questions were marked NA.

384

385

386

387

388

389

390

391

392

393

394

395

396

397

398

399

400

401

402

403

404

405

406

407

408

409

410

411

412

413

414

415

416

417

\section{Analytic Approach}

Because the topic of our study is of keen interest in both basic and applied scientific arenas, we implemented an innovative statistical approach to ensure that high standards of analytical transparency and rigor were followed to provide reliable answers to our research questions. We would have preferred to preregister our analysis protocol, a simple way to improve quality of analysis and reporting (Wagenmakers, Wetzels, Borsboom, van der Maas, \& Kievit, 2012) which has been applied to research using retrospective reports to investigate sleep outcomes (Przybylski, 2018). We, however, had accessed the data prior to our formulating our research questions, making it impossible to document our lack of foreknowledge about the data under analysis (Weston, Ritchie, Rohrer, \& Przybylski, 2019). The analyses were therefore centered around an approach called Specification Curve Analysis (SCA; Simonsohn, Simmons, \& Nelson, 2015), which has been applied recently in work both outside the area of digital media effects research (Rohrer, Egloff, \& Schmukle, 2017) and in digital media effects research specifically (Orben, Dienlin, \& Przybylski, 2019; Orben \& Przybylski, 2019a, 2019b). Instead of reporting only one result of a single possible analysis pathway, as done in most psychological studies, SCA takes into account the vast number of analytical decisions associated with the analysis of data (Steegen, Tuerlinckx, Gelman, \& Vanpaemel, 2016). These decisions quickly branch out into a vast 'garden of forking paths': a combinatorial explosion in the ways that data could have been analyzed. The choice between many different analysis pathways can skew the final results reported in a final paper (Gelman \& Loken, 2014; Orben \& Przybylski, 2019b). Using SCA, we ran and reported the results of all theoretically defensible analysis pathways, in a stepwise methodological approach which is elaborated on further below.

\section{Step 1. Preliminary analyses}

The first analytical step of our study was not related to SCA but, instead, was informative in providing a fuller picture of the available data. We first focused on examining retrospectively self-reported digital technology use, in particular by examining the correlations between retrospectively-reported digital engagement and retrospectively-reported sleep items. Examining the retrospective self-report questions allowed us to obtain an overview of what most studies in the research area are confronted with when analyzing their data. This allowed us to put our results into the context of the previous literature (e.g., Scott et al., 2019). 
418 We also examined the correlation between self-reported retrospective digital technology

419 engagement and sleep difficulties. Sleep difficulties were measured using two questions that

420 asked the adolescent participants for retrospective self-report judgements about the time it takes

421 them to fall asleep and the amount of times they awake during the night. To determine how

422 retrospective reports of digital technology use affects these judgements, we fitted a linear

423 regression predicting sleep difficulties from digital technology engagement and included all the

424 covariates also used in the SCA detailed in the next methodological subsections.

425

426

427

428

429

430

431

432

433

434

435

436

437

438

439

440

441

442

443

444

445

446

447

448

449

450

451

452

453

454

455

456

457

\section{Step 2. Identifying specifications}

The next step in the analytical process was to decide which specifications - combinations of analytical decisions or paths through the analytical 'garden of forking paths' - are theoretically defensible and should therefore be included in the SCA. First, we noted what analytical decisions needed to be taken to analyze the data, and then, for each decision, agreed on possible analytical choices that would be plausible approaches if applied to the data by other researchers interested in our research questions. For example, an analytical decision might be how to define digital engagement and the choices might include a range of possible retrospective self-report technology use items and time-use diary variables pertaining to whether technology was used and when. For the current research question, the analytical decisions included a) Whether to examine weekdays or weekend days? b) How to measure digital engagement? c) How to measure sleep? and d) What control variables to include on the basis of the existing literature? Once the possible analytical options were determined, we proceeded to step 3 of the analysis plan and implemented all possible analytical pathways in line with each possible combination of the chosen operationalizations we identified.

\section{Step 3. Implementing Specifications}

We therefore combined the various analytical decisions, and their corresponding choices, to form the specifications of our SCA. In order to account for the variety of different analytical pathways that could have been used to analyze the data, we took all the possible combinations of analytical choices and added them into a linear regression as different standardised predictors, outcomes and covariates. In other words we analysed every possible variant of the outcome variable in combination with every possible version of the predictor and every possible version of the covariates. Once we calculated all the resulting standardised regression coefficients, we examined their range of effects, while also taking note of their corresponding $p$ values, number of participants and r-squared values. We also calculated 500 bootstrapped SCAs to obtain $95 \%$ confidence intervals. All our calculations were then visualised in a graph presenting the range of possible standardised regression coefficients from all specifications and what analytical decisions caused which specified outcome. Said differently, the visualisation helps us understand what analytical decisions were most influential in determining the nature of the analytical result and what median association linked digital technology engagement and adolescent sleep. 
458 It is not possible to apply standard parametric significance tests to the results of the SCA analysis

459 due to the non-independent nature of the separate specifications. In a move away from

460 significance testing to a more in depth interpretation of the data, we decided against calculating

461 overarching non-parametric significance estimates as implemented by Orben \& Przybylski,

462 2019a and 2019b.

463

464

\section{Code Availability Statement}

465 The code used to analyze the data in the manuscript is available on the Open Science

466 Framework: https://osf.io/jhkt6/?view_only=b291a8a82f5049a5861089599e361e4a.

467

468 Results

469

470

471

\section{Step 1. Preliminary analyses}

472

We first examined the Pearson correlations between retrospective self-reported technology and

473 sleep measures (see Figure 1). These relations are especially informative because such measures

474 are normally the exclusive focus of research into digital engagement and sleep. We found that

475 there were a wide range of correlations between self-reported digital technology engagement and sleep measures ( $r=-0.03$ to -0.35 , all confidence intervals do not include zero). We also

477

478 observed correlations between self-reported retrospective digital technology engagement and

479

480 self-reported sleep difficulties: higher intensity technology users reported taking longer to fall asleep $(r=-0.12)$ and that they awake more often night $(r=-0.10)$.

481

482

483

484

485

486

487

488

489

490

491

492

493

494

495

496

497

Because these two variables are not specific to either a weekday or weekend, we could not include them in our main analyses; instead we performed two linear regressions to test their significance when accounting for all the control factors included in the main SCAs. For time taken for the adolescent to fall asleep, there was a significant negative association: standardized $\beta=-0.067, \mathrm{SE}=0.011, p<0.001$. However, for sleep disruptions during the night, there was no significant association, speaking against the generalized fears that digital technology leads to more night waking: standardized $\beta=-0.015, \mathrm{SE}=0.011, p=0.167$.

\section{Step 2. Identifying specifications}

The second part of the analysis was to determine the most useful analytical decisions necessary to address our research questions. Determining these options in turn determined the specifications we implemented in the SCA (see Table 1 for overview). We examined relations on a weekend day and weekday separately. In our analyses we could focus on three different types of sleep measures: (1) When the adolescent went to bed - higher scores indicative of an earlier bedtime - measured using retrospective self-reports, (2) When the adolescent went to bed measured using time-use diaries, and (3) Total time spent sleeping measured using retrospective self-reports. To measure technology use, we could use three possible measures taken from timeuse diaries: (1) Participation, whether the adolescent mentioned any digital technology

Peer) reviewing PDF | (2019:07:39889:2:0:NEW 12 Dec 2019) 
498

499

500

501

502

503

504

505

506

507

508

509

510

511

512

513

514

515

\section{6}

517

518

519

520

521

522

523

524

525

526

527

528

529

530

531

532

533

534

535

536

537

engagement in their daily diary, (2) Time spent, when they did mention digital technology engagement, we calculated how much time they spent on digital devices, and (3) Bedtime technology use, whether they noted down digital technology engagement 30 minutes before bedtime. We also used a self-report retrospective measure of digital technology use. While this measure asked about adolescent's digital engagement on a normal school day, we also used it as part of the weekend SCAs because its importance as a variable eclipsed this issue of scope.

The other analytical decision which needed to be accounted for was the inclusion of covariates. While previous studies (Orben \& Przybylski, 2019b, 2019a) included a "no control variables" option in their SCA models, the current study did not include this option because we did not believe the no control option is "theoretically defensible" as an analytical specification in light of simple control variables being routinely included in epidemiological and psychological research (Simonsohn et al., 2015) and those specifically found to be critical for interpreting media effects research on sleep (Przybylski, 2018). We however included either only demographics, demographics and child-level control variables, demographics and mother-level control variables, demographics and family-level control variables or all control variables in the final linear regression.

\section{Step 3. Implementing Specifications}

We identified 120 promising combinations of analytical decisions that we could use to test our research questions. The results of implementing these SCAs are visualized in Figure 2, with weekend (Panel A) and weekday (Panel B) of the SCA separated for comparison. The number of participants for each specification is displayed in Figure 3. The standardized regression coefficients that resulted from the various specifications ranged from the most positive (standardized $\beta=0.21$, specification: weekend day/using tech before bedtime/bedtime measured using time use diaries/demographic controls only) to the most negative (standardized $\beta=-0.36$, specification: weekend day/self-reported technology use/self-reported bedtime/demographic controls only). About one quarter of the specifications $(\mathrm{k}=31)$ were not statistically significant, with the weekday SCA having 12 non-significant results and the weekend SCA having 19 nonsignificant results. Furthermore, there were 8 specifications for weekdays and 10 specification for weekend days that were significant and positive. The majority of specifications, however, demonstrated negative associations between digital technology use and adolescent sleep.

To examine our two research questions probing the relation between digital technology use and sleep measures, we differentiated further between weekday and weekend days. Overall weekday digital engagement had a more negative association with sleep measures than weekend engagement (median standardized $\beta_{\text {weekday }}=-0.06$, median standardized $\beta_{\text {weekend }}=-0.03$ ). This difference is significant, as shown when applying a $t$-test comparing weekday and weekend day specifications $\left(\mathrm{t}_{60}=-5.00, \mathrm{p}<0.001\right)$. 
538 Looking at different sleep measures specifically, there were no apparent differences between

539

540

541

542

543

544

545

546

547

548

549

550

551

552

553

554

555

556

557

558

559

560

561

562

563

564

565

566

567

568

569

570

571

572

573

574

575

576

relations on weekdays or weekend days when bedtime was measured using time-use diary or retrospective self-report measures. Bedtime was delayed when adolescents used more digital technology on both weekdays (RQ1, median standardized $\beta_{\text {time-use diary }}=-0.02$, median standardized $\left.\beta_{\text {retrospective self-report }}=-0.11\right)$ and weekend days (RQ2, median standardized $\beta_{\text {time-use }}$ diary $=0.01$, median standardized $\beta_{\text {retrospective self-report }}=-0.10$ ). Interestingly, however, while bedtimes on weekdays and weekend days were equally associated with digital technology use, total time sleeping demonstrated a more negative association with digital technology use on a weekday when compared to a weekend day (median standardized $\beta_{\text {weekday }}=-0.08$, median standardized $\beta_{\text {weekend }}=-0.02$ ). This finding might support the hypothesis that negative technology effects are exacerbated on weekdays because on these days the wake-up time is static for adolescents because of school, and therefore a belated bedtime makes them lose more sleep (Scott, personal communication).

To answer our third research question, we examined different types of digital technology use measures. Most prominently, total time spent using digital technology reported via retrospective self-report questionnaires (median standardized $\beta_{\text {weekday }}=-0.23$, median standardized $\beta_{\text {weekend }}=-$ 0.13 ) and time use diaries (median standardized $\beta_{\text {weekday }}=-0.18$, median standardized $\beta_{\text {weekend }}=-$ $0.15)$ showed the most negative associations with adolescent sleep. In contrast, participation in digital technology use (median standardized $\beta_{\text {weekday }}=-0.02$, median standardized $\beta_{\text {weekend }}=$ 0.01 ) and use before bedtime (median standardized $\beta_{\text {weekday }}=-0.02$, median standardized $\beta_{\text {weekend }}$ $=-0.02$ ) showed a mix of very small negative and positive correlations, or non-significant results. The way technology use is measured is therefore important to consider in the study of these associations. In contrast, we also examined how the use of different control variables influenced the results, but there were no clear trends of some control variables shifting results in a specific direction (median standardized $\beta=-0.05$ for all specifications of control variables).

\section{Discussion}

Concerns regarding adolescents' digital engagement and its possible influence on sleep are at the forefront of popular and policy discourse (Davies et al., 2019; Viner et al., 2019). Unfortunately, the existing evidence base relies largely on imprecise measurements of digital engagement (Orben et al., 2018; Orben \& Przybylski, 2019a). A more nuanced understanding of digital engagement and its relationship to adolescent sleep grounded in transparent methods using a convergent and accurate measurement approach is necessary. This need is pronounced as most previous research does not dissociate digital engagement throughout the day from digital engagement before bedtime, most policies and draft guidance on this topic specifically make reference to digital engagement before bedtime (Davies et al., 2019; Viner et al., 2019). Guidance provided to caregivers and policymakers therefore fails to align with the evidential 
577 value of the research available. In light of this gap, our study introduces multiple improvements

578 to this important research area by diversifying measurement and improving analytical

579 transparency whilst probing digital engagement before bed. In this study we included both

580 retrospective self-report measures and time-use diary measures to examine the association

581 between digital engagement and adolescent sleep using Specification Curve Analysis. The time-

582 use diaries allow us to dissociate different measures of digital engagement: providing some of

583 the first insights based on time-use diaries into the use of digital technologies before bedtime and

584 whether this affects adolescent sleep outcomes.

585

586 In terms of our first two research questions, we investigated the association between digital

587

588

589

590

591

592

593

594

595

596

597

598

599

600

601

602

603

604

605

606

607

608

609

610

611

612

613

614

615

engagement and sleep on both weekdays and weekend days. We found evidence for negative associations between digital engagement and adolescent sleep on both weekdays $(\beta=-0.06)$ and weekend days $(\beta=-0.03)$ and found evidence that the relation on weekdays, though itself modest, is significantly more negative than the relation on weekend days. It is interesting to note, however, that there exist divergent results when examining different measures of adolescent sleep. When we examined total time spent sleeping, we found it was more negatively associated with digital technology engagement on a weekday rather than a weekend day. Such a difference was not present when we examined bedtimes: the negative association between bedtime and digital engagement was similar for both weekdays and weekend days.

This pattern could be, in part, due to wake-up times being less flexible on a weekday, where they need to wake up at a certain time for school regardless how late they stayed up at night. Because adolescents have less choice regarding their wake-up time on weekdays, they might be accruing a sleep deficit when their bedtime is forestalled by technology use. On weekend days they are often able to choose to wake up later, therefore losing less total sleep. This highlights the importance of differentiating weekdays and weekend days when investigating adolescents' technology engagement and its relationship to sleep.

It is, however, crucial to note that the effects on weekdays and weekend days were both very small, with an effect size that falls into the range of practically insignificant effects previously defined by media scholars (Ferguson, 2009; Orben \& Przybylski, 2019a; Przybylski, Orben, \& Weinstein, 2019). Whilst the standardized estimates we observe will be of interest to basic science researchers (e.g. to plan their sample sizes), these associations are possibly too small to be of great importance for policy makers or parents. While it is difficult to put the effects into context due to the many different response scales, we will do so by focusing on the hourly outcome measures we analyzed, namely the total time spent sleeping which was derived from retrospective self-report scales and the technology use before bedtime and total technology use throughout the day (derived just from time-use diaries) and all the control variables included. Doing so indicated that every additional hour of technology use throughout the day was

Peer) reviewing PDF | (2019:07:39889:2:0:NEW 12 Dec 2019) 
616 associated with 9 minutes and 17 seconds less sleep on weekdays and 3 minutes and 26 seconds 617 less sleep on weekend days.

618

619 This result conceptually replicates findings reported from a pre-registered representative cohort 620 study of American children and adolescents which estimated the impact of an hour of screen 621 time on sleep ranges 3 to 8 minutes of sleep depending on the age of the child (Przybylski, 622 2018). The modest scale of this association is similarly reflected in digital screen time before

623 bed. Results here showed that an adolescent who reported using digital technology within a half 624 an hour before bedtime reported an average of 1 minute less sleep on weekdays and 1 minute and 6253 seconds less sleep on weekend days. Framing these small effects in terms of the real-world 626 units parents and health policy-makers care about is important; they speak to the idea that 627 technology use before bed might not, in of itself, be associated with the practically significant 628

629

630

631

632

633

634

635

636

637

638

639

640

641

642 Considering the implications of the present study more broadly, the pattern of results we report

643 in this investigation makes it is increasingly evident that screen time as a concept is flawed,

644 potentially fatally so. Retrospective reports of screen time fall well short of accounting for the

645 sheer diversity of behaviors the concept and measures it stands in for. It is clear to many that 20

646 minutes on very social content, possibly co-using the technology with parents or friends, will

647 have a very different influence on a child than 20 minutes on content that can contain harmful or 648 disturbing information or images. It is therefore important for future research to incorporate a

649 greater variety of measurement into its designs, moving away from relying solely on

650

651

652

653

654

655 retrospective self-report accounts of screen time.

\section{Limitations}

While this study excels at introducing new measurement to the area of technology effects and sleep research, it is important to note three limitations that need to be taken into account before generalizing results. Firstly, while the use of time-use diaries allows us to probe more diverse 
656 measures of digital engagement, it is not known if the specific measures we are using in these 657 data necessarily contain less error than the common retrospective self-report measures. It might 658 be the case that adolescents are simply not accurate in reporting digital engagement by way of 659 time-use diaries either and how these instruments are misused is merely different. The errors in 660 the two different measurement approaches will most probably vary, making it valuable to 661 examine them in conjunction, as done in this study. That said, research specifically focused on 662 how digital engagement is recorded in time-use diaries to gauge what sort of error is associated 663 with this specific technique is needed to advance this topic of study.

664

665 Second, while our study innovated in terms of time-use diaries, the secondary nature of the data 666 meant that some of the self-reported measurements had limitations attached to them. A more

668

669

670

671

672

673

674

675

676

677

678

679

680

681

682

683

684

685

686

687

688

689

690

691

692 The widespread use of digital technologies by young people and its implication on sleep has

693 been widely discussed in both academic, caregiving and political circles. If the use of digital

694

695 which was measured on a 5-point scale but which we took to be a continuous variable for the scale of analytical clarity. The nature of the time-use diaries also made it necessary to quantify things in ways that introduced other limitations. Digital technology use before bed was operationalized as noting down use of digital technologies in the 30 minutes before the noted bedtime in the time use diaries. Again, a more continuous measure once new studies are finalized could benefit understanding further. When examining sleep using the time use diaries, we also needed to assume that the last sleep onset during the diary day was 'bedtime', which could not be the case in adolescents with disturbed sleep. Furthermore, for those adolescents who never noted a sleep onset during the diary day, we had to implement the assumption that they went to bed at midnight of that day. We therefore report further sensitivity analyses in the supplementary code. Lastly the nature of the data was limited to measuring time spent on screens and as noted above screen time is known to be a flawed measure of digital technology effects and should be replaced in future research by measures that take into account the diversity of technology use.

Finally, in an area where there is much public debate and academic discussion, it is also important to highlight the correlational nature of this study. The results should therefore not be used to make directional or causal conjectures. As there are currently no multi-wave time-use diary studies that would have allowed us to examine diverse measures of digital engagement, this limitation is a clear tradeoff as we cannot examine longitudinal within-person effects. In future, more longitudinal data collection with diverse measurement methods would allow for directional conjectures to be made.

\section{Conclusions} screens either throughout the day or before bedtime undermines sleep quality, duration, or causes a shift in bedtimes, this needs to be noted and addressed quickly. In this study we found that continuous measure would have also benefited our report of adolescent's self-reported bedtimes, 
696 digital technology use is negatively correlated with sleep measured on both weekends and 697 weekdays, however these correlations were small. While there were not many pronounced

698 differences between the relations on a weekday compared to a weekend day: weekdays showed a

699

700

701

702

703

704

705

706

707

708

709

710

711

712

713

714

715

716

717

718

719

720

721

722

723

724

725

726

727

728

729

730

731

732

733

734

735

736

more negative association between digital technology use and total time spent sleeping, something that could hint at the rigidity of waking times on school days. It is interesting to note that no clear associations were found when examining digital technology use before bedtime specifically, and that the negative associations were driven by retrospective accounts of digital technology use or total time spent using digital technologies as reported in time use diaries. This highlights the importance of thinking about how digital technology use is measured, even though in the current scientific landscape the scope and nature of measurement are often overlooked (Flake \& Fried, 2019). Diversifying the measurement of digital engagement is crucial to test the robustness of the effects found in previous research. Our study using time-use diaries in conjunction with transparent analysis methods marks a valuable first step towards enabling such measurement diversification, allowing researchers to examine different measures of digital engagement and sleep that have long been neglected in a new light. In future, policy makers, academics and industry need to collaborate in a more concentrated effort to further improve the measurement of digital engagement, for example by finding ways to share individual trace data currently housed on servers of social media and technology companies. Only when research can fully remove itself from the problematic self-report measurement of digital technology use, will it be able to provide clear insights into how digital engagement is affecting current adolescents and, ultimately, the whole global population.

\section{Acknowledgements}

Centre for Longitudinal Studies, UCL Institute of Education collected MCS and the UK Data Archive/UK Data Service provided the data; They bear no responsibility for its analysis or interpretation. The Millennium Cohort Study is funded by grants from Economic and Social Research Council. Amy Orben an Andrew Przybylski were funded by a grant from Barnardo's UK; Amy Orben received funding from Emmanuel College, University of Cambridge, Andrew Przybylski received funding from the Huo Family Foundation.

\section{References}

Andrews, S., Ellis, D. A., Shaw, H., \& Piwek, L. (2015). Beyond Self-Report: Tools to Compare Estimated and Real-World Smartphone Use. PloS ONE, 10(10), e0139004. https://doi.org/10.1371/journal.pone.0139004

Beebe, D. W. (2011). Cognitive, behavioral, and functional consequences of inadequate sleep in children and adolescents. Pediatric Clinics of North America, 58(3), 649-665. https://doi.org/10.1016/j.pcl.2011.03.002

Boase, J., \& Ling, R. (2013). Measuring Mobile Phone Use: Self-Report Versus Log Data. Journal of Computer-Mediated Communication, 18, 508-519.

PeerJ reviewing PDF | (2019:07:39889:2:0:NEW 12 Dec 2019) 
737

738

739

740

741

742

743

744

745

746

747

748

749

750

751

752

753

754

755

756

757

758

759

760

761

762

763

764

765

766

767

768

769

770

771

772

773

774

775

776

777

778

779

780

781

782

https://doi.org/10.1111/jcc4.12021

Cain, N., \& Gradisar, M. (2010). Electronic media use and sleep in school-aged children and adolescents: A review. Sleep Medicine, 11, 735-742. https://doi.org/10.1016/j.sleep.2010.02.006

Constine, J. (2019). Facebook will shut down its spyware VPN app Onavo. TechCrunch.

David, M. E., Roberts, J. A., \& Christenson, B. (2018). Too Much of a Good Thing: Investigating the Association between Actual Smartphone Use and Individual Well-Being. International Journal of Human-Computer Interaction, 34(3), 265-275. https://doi.org/10.1080/10447318.2017.1349250

Davies, S. C., Atherton, F., Calderwood, C., \& McBride, M. (2019). United Kingdom Chief Medical Officers' commentary on 'Screen-based activities and children and young people's mental health and psychosocial wellbeing: a systematic map of reviews. " Retrieved from https://assets.publishing.service.gov.uk/government/uploads/system/uploads/attachment_dat a/file/777026/UK_CMO_commentary_on_screentime_and_social_media_map_of_reviews. pdf

Ferguson, C. J. (2009). An effect size primer: A guide for clinicians and researchers. Professional Psychology: Research and Practice, 40(5), 532-538. https://doi.org/10.1037/a0015808

Flake, J. K., \& Fried, E. I. (2019). Measurement Schmeasurement: Questionable Measurement Practices and How to Avoid Them. Preprint. https://doi.org/10.31234/OSF.IO/HS7WM

Gelman, A., \& Loken, E. (2014). The garden of forking paths: Why multiple comparisons can be a problem, even when there is no "fishing expedition" or "p-hacking" and the research hypothesis was posited ahead of time. New York, NY: Department of Statistics, Columbia University. https://doi.org/dx.doi.org/10.1037/a0037714

Hammer, B. (2012). Statistical Models for Time Use Data: An Application to Housework and Childcare Activities Using the Austrian Time Use Surveys from 2008 and 1992. University of Vienna. Retrieved from http://www.ntaccounts.org/doc/repository/Statistical Models for Time Use Data - An Application....pdf

Harbard, E., Allen, N. B., Trinder, J., \& Bei, B. (2016). What's Keeping Teenagers Up? Prebedtime Behaviors and Actigraphy-Assessed Sleep over School and Vacation. Journal of Adolescent Health, 58, 426-432. https://doi.org/10.1016/j.jadohealth.2015.12.011

Ipsos MORI. (2016). Millennium Cohort Study Sixth Sweep (MCS6) Time Use Diary Documentation.

Levenson, J. C., Shensa, A., Sidani, J. E., Colditz, J. B., \& Primack, B. A. (2017). Social media use before bed and sleep disturbance among young adults in the United States: A nationally representative study. Sleep, 40(9), 1-7. https://doi.org/10.1093/sleep/zsx113

Masur, P. K. (2019). Situational Privacy and Self-Disclosure (1st ed.). Springer International Publishing. https://doi.org/10.1007/978-3-319-78884-5

Ofcom. (2019). Children and parents: media use and attitudes report 2018. Retrieved from https:/www.ofcom.org.uk/research-and-data/media-literacy-research/childrens/childrenand-parents-media-use-and-attitudes-report-2018

Orben, A., Dienlin, T., \& Przybylski, A. K. (2019). Social media's enduring effect on adolescent life satisfaction. Proceedings of the National Academy of Sciences of the United States of America, 116(21), 10226-10228. https://doi.org/10.1073/pnas.1902058116

Orben, A., Etchells, P. J., \& Przybylski, A. K. (2018). Three problems with the debate around screen time. The Guardian. 
783

784

785

786

787

788

789

790

791

792

793

794

795

796

797

798

799

800

801

802

803

804

805

806

807

808

809

810

811

812

813

814

815

816

817

818

819

820

821

822

823

824

825

826

827

828

Orben, A., \& Przybylski, A. K. (2019a). Screens, Teens, and Psychological Well-Being: Evidence From Three Time-Use-Diary Studies. Psychological Science, 30(5), 682-696. https://doi.org/10.1177/0956797619830329

Orben, A., \& Przybylski, A. K. (2019b). The association between adolescent well-being and digital technology use. Nature Human Behaviour, 3(2), 173-182. https://doi.org/10.1038/s41562-018-0506-1

Orzech, K. M., Grandner, M. A., Roane, B. M., \& Carskadon, M. A. (2016). Digital media use in the $2 \mathrm{~h}$ before bedtime is associated with sleep variables in university students. Computers in Human Behavior, 55, 43-50. https://doi.org/10.1016/J.CHB.2015.08.049

Owens, J. (2014). Insufficient Sleep in Adolescents and Young Adults: An Update on Causes and Consequences. Pediatrics, 134(3), e921-e932. https://doi.org/10.1542/peds.2014-1696

Parkes, A., Sweeting, H., Wight, D., \& Henderson, M. (2013). Do television and electronic games predict children's psychosocial adjustment? Longitudinal research using the UK Millennium Cohort Study. Archives of Disease in Childhood, 98(5), 341-348. https://doi.org/10.1136/archdischild-2011-301508

Przybylski, A. K. (2018). Digital Screen Time and Pediatric Sleep: Evidence from a Preregistered Cohort Study. https://doi.org/10.1016/j.jpeds.2018.09.054

Przybylski, A. K., Orben, A., \& Weinstein, N. (2019). How Much Is Too Much? Examining the Relationship Between Digital Screen Engagement and Psychosocial Functioning in a Confirmatory Cohort Study. Journal of the American Academy of Child and Adolescent Psychiatry, O(0). https://doi.org/10.1016/j.jaac.2019.06.017

Reis, H. T., Gable, S. L., \& Maniaci, M. R. (2013). Methods for Studying Everyday Experience in Its Natural Context. In H. T. Reis \& C. M. Judd (Eds.), Handbook of research methods in social and personality psychology (Second, p. 744). New York: Cambridge University Press.

Robinson, J. R. (1985). The Validity and Reliability of Diaries versus Alternative Time Use Measures. In F. Thomas \& F. P. Sanford (Eds.), Time, Goods and Well-Being (pp. 33-62). Ann Arbor, MI: Institute for Social Research, University of Michigan.

Rohrer, J. M., Egloff, B., \& Schmukle, S. C. (2017). Probing Birth-Order Effects on Narrow Traits Using Specification-Curve Analysis. Psychological Science, 28(12), 1821-1832. https://doi.org/10.1177/0956797617723726

Rohrer, J. M., \& Lucas, R. E. (2018). Only so Many Hours : Correlations between Personality and Daily Time Use in a Representative German Panel. Collabra: Psychology, 4(1), 1-10. https://doi.org/https://doi.org/10.1525/collabra.112

Scharkow, M. (2016). The Accuracy of Self-Reported Internet Use-A Validation Study Using Client Log Data. Communication Methods and Measures, 10(1), 13-27. https://doi.org/10.1080/19312458.2015.1118446

Schwarz, N., \& Oyserman, D. (2001). Asking Questions About Behavior: Cognition, Communication, and Questionnaire Construction. American Journal of Evaluation, 22(2), 127-160. https://doi.org/10.1177/109821400102200202

Scott, H., Biello, S. M., \& Cleland, H. (2018). Identifying drivers for bedtime social media use despite sleep costs : the adolescent perspective. Preprint. https://doi.org/https://doi.org/10.31234/osf.io/2xb36

Scott, H., Biello, S. M., \& Woods, H. (2019). Social media use and adolescent sleep outcomes: cross-sectional findings from the UK Millennium Cohort Study. Preprint. https://doi.org/10.31234/OSF.IO/Z7KPF

Peer) reviewing PDF | (2019:07:39889:2:0:NEW 12 Dec 2019) 
829

830

831

832

833

834

835

836

837

838

839

840

841

842

843

844

845

846

847

848

849

850

851

852

853

854

855

856

857

858

859

860

861

862

863

864

865

866

Simonsohn, U., Simmons, J. P., \& Nelson, L. D. (2015). Specification Curve: Descriptive and inferential statistics on all reasonable specifications. SSRN Electronic Journal. https://doi.org/10.2139/ssrn.2694998

Steegen, S., Tuerlinckx, F., Gelman, A., \& Vanpaemel, W. (2016). Increasing Transparency Through a Multiverse Analysis. Perspectives on Psychological Science, 11(5), 702-712. https://doi.org/10.1177/1745691616658637

University of London, Institute for Education, C. for L. S. (2017). Millennium Cohort Study: Sixth Survey, 2015. UK Data Service. https://doi.org/http://doi.org/10.5255/UKDA-SN8156-2

Vanden Abeele, M., Beullens, K., \& Roe, K. (2013). Measuring mobile phone use: Gender, age and real usage level in relation to the accuracy and validity of self-reported mobile phone use. Mobile Media \& Communication, 1(2), 213-236. https://doi.org/10.1177/2050157913477095

Verduyn, P., Lee, D. S., Park, J., Shablack, H., Orvell, A., Bayer, J., ... Kross, E. (2015). Passive Facebook usage undermines affective well-being: Experimental and longitudinal evidence. Journal of Experimental Psychology: General, 144(2), 480-488. https://doi.org/10.1037/xge0000057

Viner, R., Davie, M., \& Firth, A. (2019). The health impacts of screen time: a guide for clinicians and parents. Retrieved from https://www.rcpch.ac.uk/sites/default/files/201812/rcpch_screen_time_guide_-_final.pdf

Wagenmakers, E.-J., Wetzels, R., Borsboom, D., van der Maas, H. L. J., \& Kievit, R. A. (2012). An Agenda for Purely Confirmatory Research. Perspectives on Psychological Science, 7(6), 632-638. https://doi.org/10.1177/1745691612463078

Weston, S. J., Ritchie, S. J., Rohrer, J. M., \& Przybylski, A. K. (2019). Recommendations for Increasing the Transparency of Analysis of Preexisting Data Sets. Advances in Methods and Practices in Psychological Science, 251524591984868. https://doi.org/10.1177/2515245919848684

Williams, J. S., Greene, S., Doyle, E., Harris, E., Layte, R., McCoy, S., \& McCrory, C. (2009). Growing Up in Ireland National Longitudinal Study of Children: The lives of 9-year-olds. Dublin: Office of the Minister for Children and Youth Affairs.

Wonneberger, A., \& Irazoqui, M. (2017). Explaining Response Errors of Self-Reported Frequency and Duration of TV Exposure Through Individual and Contextual Factors. Journalism \& Mass Communication Quarterly, 94(1), 259-281. https://doi.org/10.1177/1077699016629372

Woods, H. C., \& Scott, H. (2016). \#Sleepyteens: Social media use in adolescence is associated with poor sleep quality, anxiety, depression and low self-esteem. Journal of Adolescence, 51, 41-49. https://doi.org/10.1016/j.adolescence.2016.05.008 


\section{Table $\mathbf{1}$ (on next page)}

Table showing the four different analytical decisions that needed to be taken to analyze the data

The decisions were: what day of the week to analyze, how to define sleep, how to define technology use, and which control variables to include. Each analytical decision had multiple analytical options that could have been taken. Each unique combination of different analytical options that could be implemented to analyze the data is a specification. In total, this study encompassed 120 defined specifications. 
1 Table 1: Table showing the four different analytical decisions that needed to be taken to analyze the

2 data

3 The decisions were: what day of the week to analyze, how to define sleep, how to define technology

4 use, and which control variables to include. Each analytical decision had multiple analytical options that

5 could have been taken. Each unique combination of different analytical options that could be

6 implemented to analyze the data is a specification. In total, this study encompassed 120 defined

7 specifications.

\begin{tabular}{lllll}
\hline $\begin{array}{l}\text { Analytical } \\
\text { Decision }\end{array}$ & Day of the Week & Sleep & Technology Use & Control Variables \\
\hline $\begin{array}{l}\text { Analytical } \\
\text { Options }\end{array}$ & Weekday & $\begin{array}{l}\text { Bedtime } \\
\text { (retrospective) }\end{array}$ & $\begin{array}{l}\text { Participation (time- } \\
\text { use diary) }\end{array}$ & Demographics \\
& Weekend day & $\begin{array}{l}\text { Bedtime (time-use } \\
\text { diary) }\end{array}$ & $\begin{array}{l}\text { Before bedtime } \\
\text { (time-use diary) }\end{array}$ & $\begin{array}{l}\text { Demographics + } \\
\text { Child-Level }\end{array}$ \\
& $\begin{array}{l}\text { Total time sleeping } \\
\text { (retrospective) }\end{array}$ & $\begin{array}{l}\text { Total time spent } \\
\text { (time-use diary) }\end{array}$ & $\begin{array}{l}\text { Demographics + } \\
\text { Mother-Level }\end{array}$ \\
& & $\begin{array}{l}\text { Total time spent } \\
\text { (retrospective) }\end{array}$ & $\begin{array}{l}\text { Demographics }+ \\
\text { Family-Level }\end{array}$ \\
& & & $\begin{array}{l}\text { All Control } \\
\text { Variables }\end{array}$ \\
\hline
\end{tabular}

8 


\section{Figure 1}

Correlation matrix showing the correlation between various self-report sleep and digital engagement measures.

Red indicates negative correlations while blue indicates positive correlations.

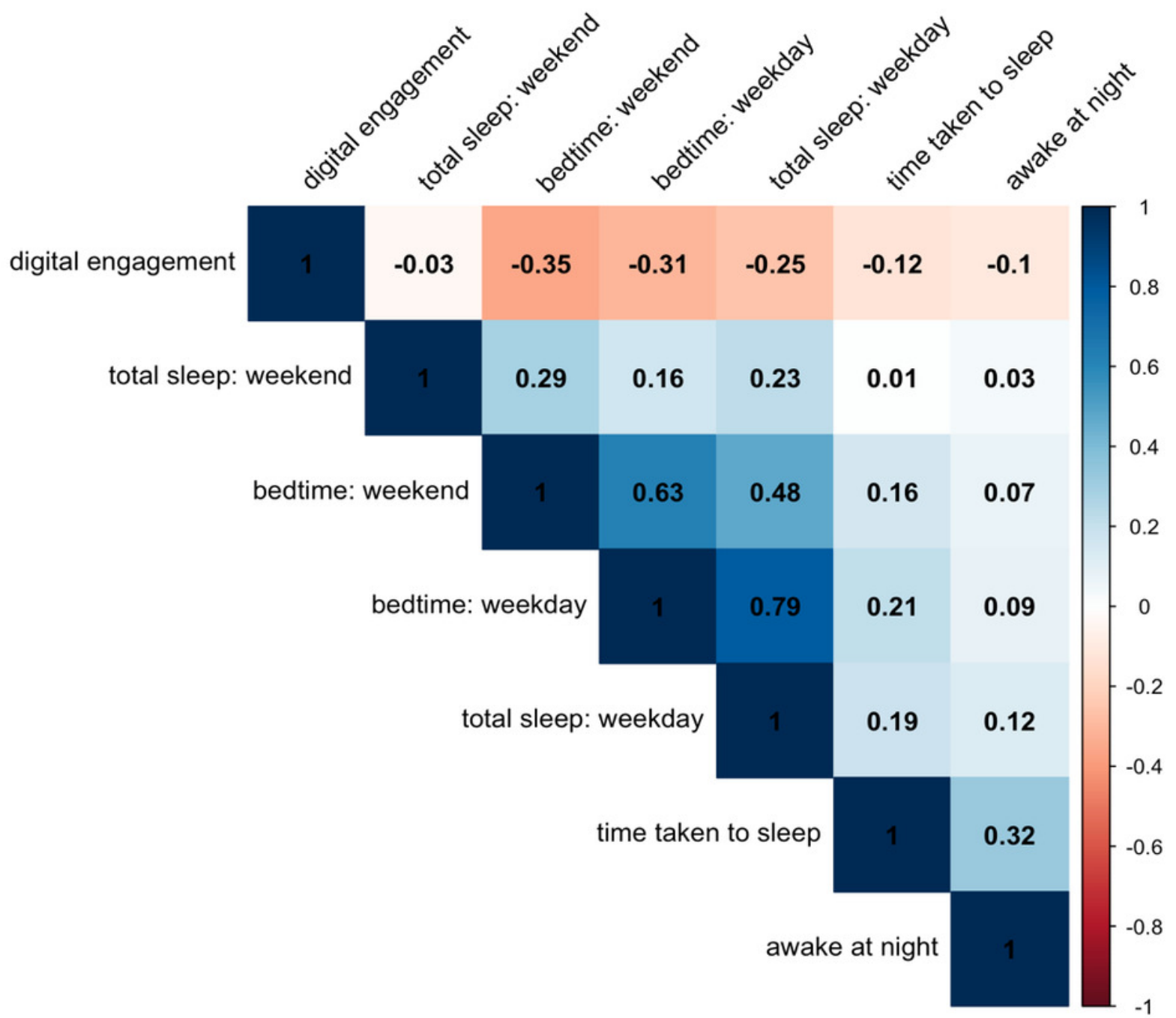




\section{Figure 2}

\section{Specification Curve Analysis}

SCA showing the results of the two separate SCAs for weekdays (Panel A; 60 specifications) and weekend days (Panel B; 60 specifications) with standardized regression coefficients presented in ranked order ranging from those results with the most negative regression associations to those with the most positive ones. In the top half of the graph the resulting standardized regression coefficient is shown. In the bottom half of the graph one can read off the analytical decisions that constitute the specification that results in the corresponding standardized regression coefficient $(*=$ analytical variables calculated using time-use diaries). Teal dots represent statistically significant specifications ( $p$-value $<0.05$ ) while pink dots represent non- significant specifications ( $p$-value $>0.05$ ).
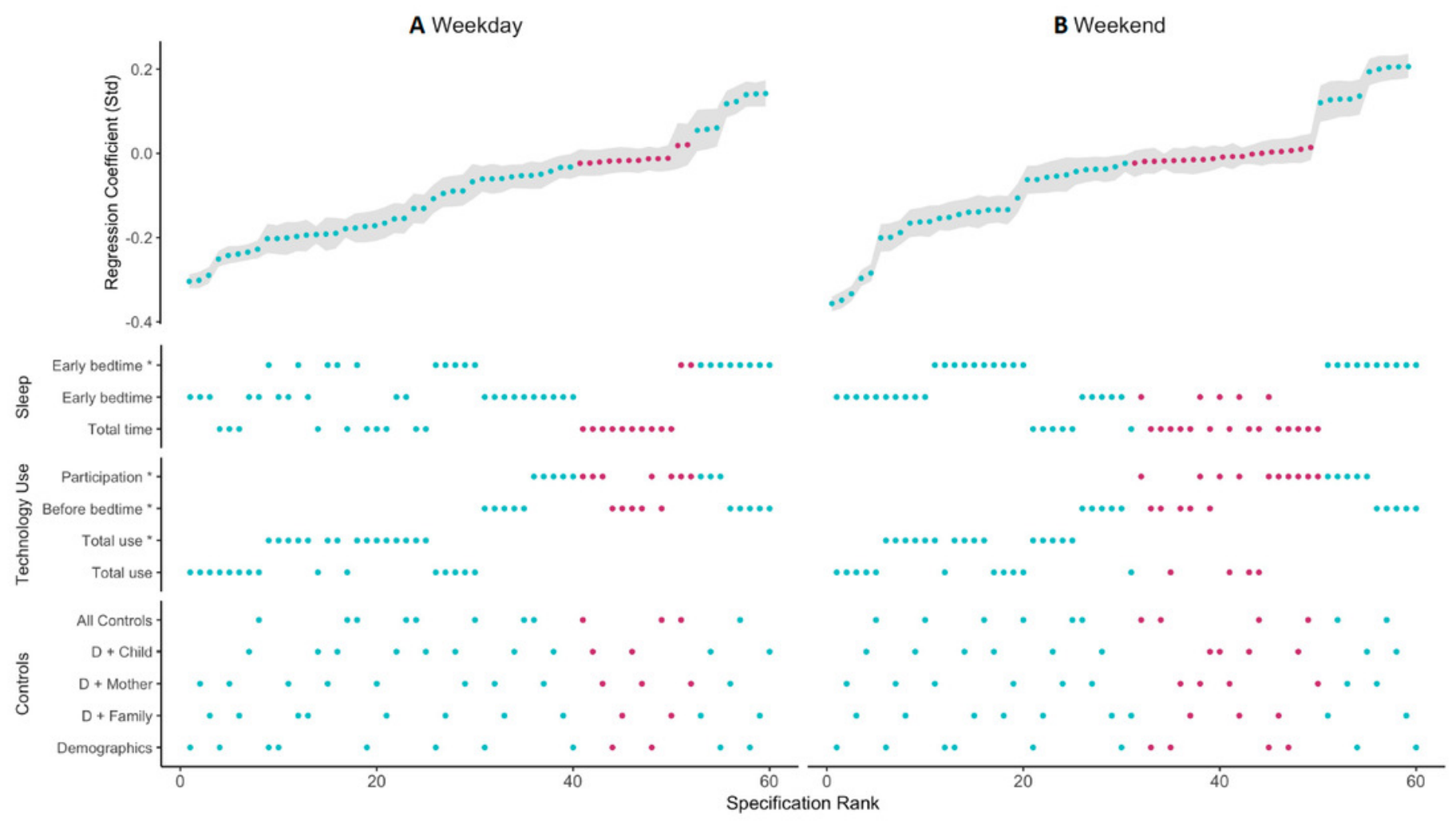
Figure 3

Number of participants included in each specification

Each point shows the amount of participants included in the analysis of each specification visualised in Figure 2 


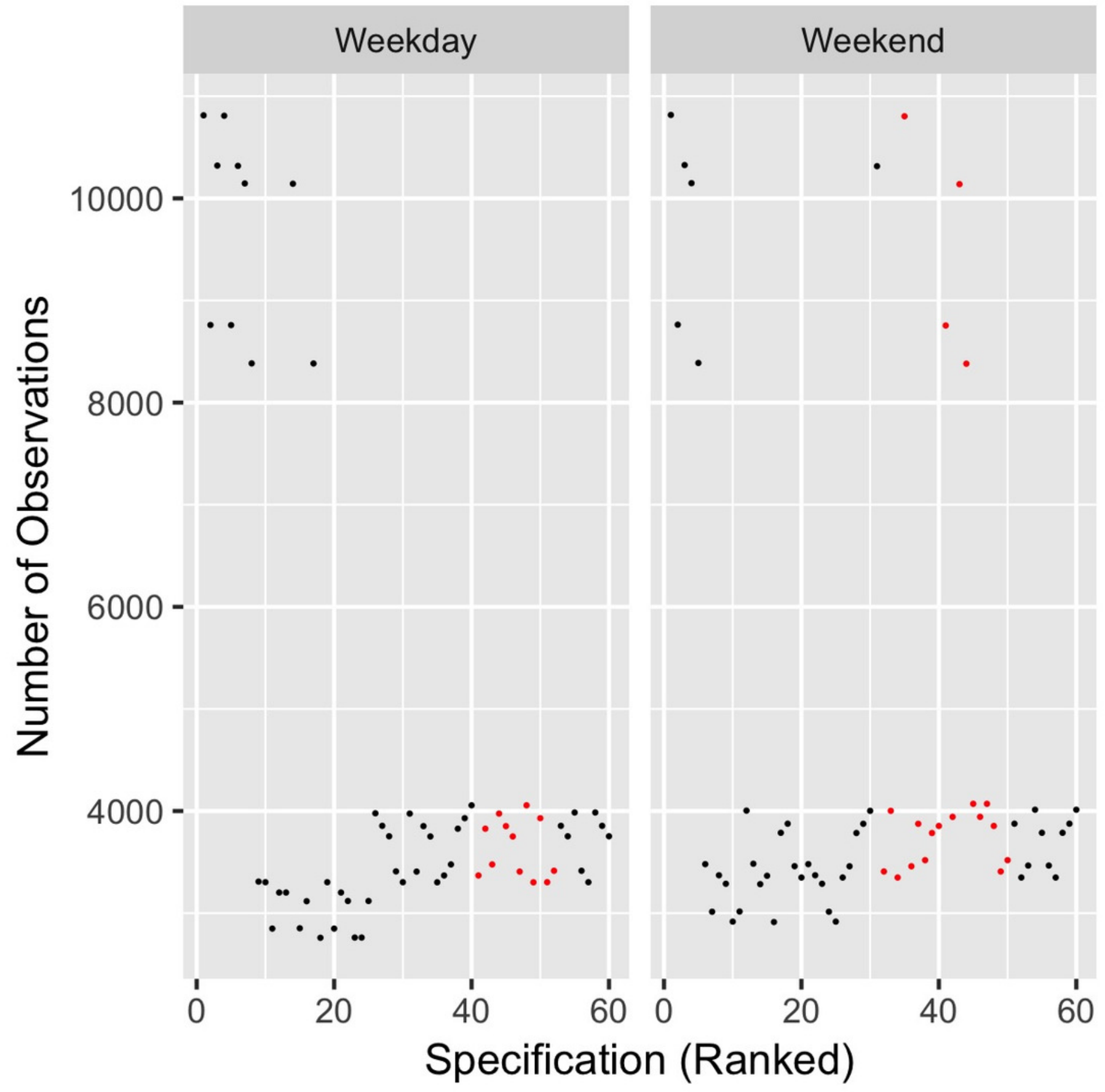

